\title{
Development and Characterization of Solid Self-emulsifying Drug Delivery System of Cilnidipine
}

\author{
Suparna Sacchit Bakhle* and Jasmine Gev Avari \\ Pharmaceutics Division, Department of Pharmaceutical Sciences, R.T.M Nagpur University; Mahatma Jyotiba Fuley \\ Shaikshanik Parisar, Amravati Road, Nagpur-440033, Maharashtra, India.
}

Received April 25, 2014; accepted March 30, 2015

The present investigations highlight the development of solid self-emulsifying drug delivery system (solid-SEDDS) for improved oral delivery of the poorly water-soluble drug; cilnidipine. Liquid SEDDS of the drug were formulated using Capryol 90 as the oil phase, Tween 80 as the surfactant, and Transcutol HP as the co-surfactant after screening various vehicles. The prepared systems were characterized for selfemulsification time, robustness to dilution, \% transmittance, globule size, drug release, and thermodynamic stability. Ternary phase diagrams were plotted to identify the area of microemulsification. The optimized liquid SEDDS was transformed into a free-flowing powder using Neusilin US2 as the adsorbent. Solid selfemulsifying powder retained the self-emulsifying property of the liquid SEDDS. Differential scanning calorimetric, X-ray powder diffraction studies revealed the possibility of transformation of the crystalline form of the drug to the amorphous form in the SEDDS prepared with the carrier. The morphology of solid-SEDDS from scanning electron microscopy studies demonstrated the presence of spherical, granular particles indicating good flowing ability. Dissolution studies revealed enhanced dissolution of the drug from the solid system compared with the pure drug and its marketed formulation. Similarly, the in vitro absorption profile of the drug from the formulated SEDDS was significantly higher compared with pure drug. Thus it can be concluded that solid-SEDDS, amenable for development of solid dosage form, can be successfully developed using Neusilin US2 with the potential of enhancing the solubility, dissolution rate, and bioavailability of the drug.

Key words cilnidipine; solid self-emulsifying drug delivery system (SEDDS); adsorption; crystallinity; droplet size; drug release

Poorly water-soluble drug candidates often emerge from contemporary drug discovery programs, and present formulators with considerable technical challenges. The absorption of such compounds when presented in the crystalline state to the gastrointestinal tract is typically dissolution rate-limited, and the drugs are typically Biopharmaceutical Classification System (BCS) class II or class IV compounds. ${ }^{1)}$ The interests on lipid-based drug delivery systems (LBDDS) have increased over the past two decades as a function of identification of these pharmaceutically difficult candidates, and increased even further after successful launch of lipid-based oral pharmaceutical products, including in particular cyclosporine A, marketed as Sandimmune ${ }^{\mathrm{TM}}$ and Neoral ${ }^{\mathrm{TM}}{ }^{2}$ )

Lipid based drug delivery system such as self-emulsifying drug delivery systems (SEDDS) are mixtures of oils and surfactants, ideally isotropic, sometimes including cosolvents, which emulsify under conditions of gentle agitation, similar to those which would be encountered in the gastrointestinal tract. Hydrophobic drugs can often be dissolved in SEDDS allowing them to be encapsulated as unit dosage forms for peroral administration. ${ }^{3-5)}$ When such a system is released in the lumen of the gastrointestinal tract, it disperses to form a fine emulsion (micro/nano) with the aid of gastrointestinal (GI) fluid. This leads to in situ solubilization of drug that can subsequently be absorbed by lymphatic pathways, bypassing the hepatic first pass effect. $\left.{ }^{6}\right)$ SEDDS formulations are viscous liquids and thus marketed usually in the form of soft gelatin capsules, which have some drawbacks in the manufacturing process such as difficulty in process control, leakage of the encapsulated components, high production cost, and lower stability. ${ }^{7)}$ To address these problems, several attempts have been made to transform liquid SEDDS into solid dosage forms using solid carriers or adsorbents. The solid forms of SEDDS are able to offer the advantages of SEDDS in combination with those of solid dosage forms such as production reproducibility and improved stability when they would lead to the formation of fine or microemulsion at a similar rate exhibited by liquid SEDDS. ${ }^{8)}$

With this background in mind the present research work focuses on the development of solid self-emulsifying drug delivery system (solid-SEDDS) for the poorly water soluble drug, cilnidipine (CND). CND is a unique $\mathrm{Ca}^{2+}$ channel blocker with an inhibitory action on the sympathetic N-type $\mathrm{Ca}^{2+}$ channels, which is used for patients with hypertension. ${ }^{9}$ The drug is practically insoluble in aqueous media and exhibits a low oral bioavailability or limited clinical efficacy. ${ }^{10}$ ) Attempts have been made by the researchers to enhance solubility and bioavailability of the drug through solid dispersions and inclusion complexes. ${ }^{10-12)}$ The suitability of SEDDS as a delivery system for improved oral delivery of CND has not been explored till date. Hence the present study was intended to design and develop SEDDS for the drug with the objective of enhancing the solubility, dissolution rate and ultimately the bioavailability of the drug. Since the liquid formulations of SEDDS are not desirable as mentioned earlier further it was envisaged in the current investigations to transform liquid SEDDS of the drug into a free flowing powder amenable for the development of a solid dosage form like capsules or tab- 
lets. Neusilin US2, a granular and highly porous material with surface area 110 to $300 \mathrm{~m}^{2} / \mathrm{g}$ and adsorption capacity of 1.3 to $3.1 \mathrm{~mL} / \mathrm{g}$ was used as the adsorbent in the present case. ${ }^{13)}$

\section{Experimental}

Materials CND was obtained as a generous gift from Macleods Pharmaceuticals Ltd., Mumbai, India. Captex 500, Captex 355, Capmul MCM were kindly supplied by Abitec Corporation, Janesville, U.S.A. while Capryol 90, Transcutol HP, Labrafil M 1944, Lauroglycol, Labrafac Lipophile WL 1349 and Labrasol were gifted by Gattefosse Ltd., Mumbai, India. Neusilin US2 was supplied by Gangwal Chemicals, Mumbai, India. Acrysol EL 135 and Acrysol K 140 were obtained as gift samples from Corel Pharma Chem, Ahmedabad, India. Tween 80, polyethylene glycol (PEG) 400 and propylene glycol were purchased from Merck (Mumbai, India). All the excipients and reagents were of analytical grade and double distilled water was freshly prepared when ever required throughout the study.

\section{Methods}

Solubility Studies

Solubility studies were carried by placing an excess amount of CND in a screw capped vials containing $2 \mathrm{~mL}$ of vehicles (oils, surfactants and co-surfactants). The suspensions of vehicles were heated on a water bath at $40^{\circ} \mathrm{C}$ to facilitate the solubilization using vortex mixer. The suspensions were then continuously agitated on a rotary shaker for $48 \mathrm{~h}$ at ambient temperature. After reaching equilibrium the samples were centrifuged at $5000 \mathrm{rpm}$ for $15 \mathrm{~min}$ and the supernatant was taken, filtered through $0.45 \mu \mathrm{m}$ membrane filter. The filtrates were suitably diluted with methanol and analyzed spectrophotometrically for the dissolved drug at $240 \mathrm{~nm}^{14)}$ Blank was prepared by dissolving respective vehicles in methanol with same dilution as for the samples. The experiment was performed in triplicate and results were represented as mean value (in $\mathrm{mg} / \mathrm{mL}$ ) \pm standard deviation (S.D.).

Preliminary Screening of Surfactants

The surfactants were screened for emulsification ability as per method reported in literature. ${ }^{15)}$ Oil was mixed with each surfactant in 1:1 ratio and vortexed for $5 \mathrm{~min}$ to ensure proper mixing. This mixture, $50 \mathrm{mg}$, was weighed and diluted to $50 \mathrm{~mL}$ with double-distilled water to obtain emulsion. The emulsions were observed for physical appearance, clarity and phase separation. The emulsions were left undisturbed for $2 \mathrm{~h}$ and analyzed for transmittance at $650 \mathrm{~nm}$ on UV spectrophotometer (Jasco V-630, Japan) using double distilled water as blank. The experiment was performed in triplicate.

Preliminary Screening of Co-surfactants

The turbidimetric method described under preliminary screening of surfactants was used to screen the co-surfactants. During screening, oil/surfactant/cosurfactant ratio was kept constant as $3: 2: 1$. The experiment was performed in triplicate.

Construction of Pseudoternary Phase Diagrams

The selected oil, surfactant, co-surfactant on the basis of solubility and preliminary screening studies were used to develop pseudoternary phase diagrams using water titration method. ${ }^{16)}$ The surfactant and co-surfactant were mixed $\left(S_{\text {mix }}\right)$ in various ratios $(1: 1,2: 1$ and $1: 2 \mathrm{w} / \mathrm{w})$ for plotting the phase diagrams. For each phase diagram, a series of oil $/ S_{\text {mix }}$ mixtures were prepared at all nine combinations $(1: 9,2: 8,3: 7$,
$4: 6,5: 5,6: 4,7: 3,8: 2,9: 1)$ and titrated with water to identify the microemulsion region. The total water consumed was noted in terms of $\mathrm{w} / \mathrm{w}$ and during titration of oil- $S_{\text {mix }}$ ratio observations were made for phase clarity. The concentration of water at which turbidity-to-transparency and transparencyto-turbidity transitions occur were derived from the weight measurements. These values were used to determine the boundaries of the microemulsion region corresponding to the selected value of oil and $S_{\text {mix }}$ ratio. Phase diagrams were constructed using CHEMIX school software, version 3.6.

Preparation of Liquid SEDDS

A series of SEDDS formulations were prepared with varying ratios of oil (20-40\%), surfactant (30-70\%) and cosurfactant (10-50\%). A single dose of CND (10 mg) was incorporated in all formulations. The total weight of the formulations was kept at $130 \mathrm{mg}$. The formulations were prepared by dissolving the drug in oil followed by addition of surfactant and co-surfactant in glass vials. The resulting mixtures were stirred continuously by vortex mixing followed by sonication for few minutes to obtain a homogenous isotropic mixture. The SEDDS formulations were stored at ambient temperatures until further use.

\section{Characterization of SEDDS}

Visual Assessment of Self-Emulsification A visual test to assess the self-emulsification properties reported by Talegaonkar et al. was modified and adopted in the present study. In this method, a unit dose of the formulation was introduced into $250 \mathrm{~mL}$ of water in a glass beaker that was maintained at $37 \pm 0.5^{\circ} \mathrm{C}$ and the contents mixed gently using a magnetic stirrer. The tendency to emulsify spontaneously and progress of emulsion droplets were observed. On the basis of dispersibility, appearance and time required to emulsify SEDDS were categorized in "Grade A," "Grade B" and "Grade C."17) All the trials were carried out in triplicate with similar observations being made between repeats.

Effect of $\mathrm{pH}$ and Robustness to Dilution

Formulations were subjected to 50, 100, 250 and 1000 fold dilution with distilled water, $\mathrm{pH} 1.2$ and $\mathrm{pH} 6.8$ buffer. The resultant diluted emulsions were monitored for any physical changes such as (coalescence of droplets, precipitation or phase separation) after $24 \mathrm{~h}$ storage. ${ }^{18)}$

\section{$\%$ Transmittance}

The SEDDS were reconstituted with distilled water and the resulting microemulsions were observed visually for any turbidity. Thereafter its \% transmittance was measured at $650 \mathrm{~nm}$ using UV-visible (Vis) spectrophotometer (Jasco V-630, Japan) against distilled water as the blank. The studies were conducted after 100 times dilution.

Droplet Size Analysis

The mean droplet size (SMD) and polydispersity index (PDI) of the formulations were determined by photon correlation spectroscopy using nanosizer (Nanophox NX0088, Sympatec, Germany). Each formulation was diluted with filtered $(0.45 \mu \mathrm{m}$, Millipore) double distilled water before analysis. Size analysis was carried at $25^{\circ} \mathrm{C}$ with an angle of detection of $90^{\circ}$.

\section{Cloud Point}

The cloud point measurement was carried out for the formulations as reported earlier. ${ }^{19)}$ The formulation was diluted up to 100 folds with distilled water and kept in a water bath which was maintained at a temperature of $25^{\circ} \mathrm{C}$ with gradual 
increase of temperature at a rate of $5^{\circ} \mathrm{C} / \mathrm{min}$ and the corresponding cloud point temperatures were read at first sign of turbidity by visual observation.

Thermodynamic Stability

The formulations were subjected to heating cooling cycle $\left(4^{\circ} \mathrm{C}\right.$ and $\left.45^{\circ} \mathrm{C}\right)$ and freeze thaw cycle $\left(-21^{\circ} \mathrm{C}\right.$ and $\left.+25^{\circ} \mathrm{C}\right)$ with storage at each temperature of not less than $48 \mathrm{~h}$. For centrifugation stress, the formulations were centrifuged at $3500 \mathrm{rpm}$ for $15 \mathrm{~min}$ and the extent of phase separation was monitored. ${ }^{20)}$

\section{Drug Release Studies}

In vitro drug release studies were performed using a modified dialysis technique. ${ }^{21)}$ Initially the dialysis tubing $(7 \mathrm{~cm}$ in length) was soaked in the dialysis medium for $12 \mathrm{~h}$ at room temperature which was treated at $40^{\circ} \mathrm{C}$ before start of the experiment. The SEDDS formulation (equivalent to $10 \mathrm{mg}$ Cilnidipine) was diluted with $1 \mathrm{~mL}$ dialysis medium and placed in dialysis tubing (Hi media membrane, Mumbai, cut off 12000-14000Da) clamped on both sides. The secured dialysis tube was allowed to rotate freely in the dissolution vessel of USP XXIV type-II dissolution apparatus (Electrolab TDT-06 T, Mumbai, India) containing $500 \mathrm{~mL}$ of $\mathrm{pH} 1.2$ buffer as dialysis medium at $37 \pm 0.5^{\circ} \mathrm{C}$ and stirred at $50 \mathrm{rpm}$. An aliquot of $5 \mathrm{~mL}$ was withdrawn at predetermined time intervals and filtered through $0.45 \mu \mathrm{m}$ filter. The withdrawn volume was replenished immediately with equal volume of fresh medium in order to keep total volume constant and maintain sink conditions. The concentration of CND in the filtrate was analyzed using UV spectrophotometer at $240 \mathrm{~nm}$. The blank SEDDS without drug was processed similarly and used as a reference to avoid interference from the formulation components, if any. Each release study was performed in triplicate. The data was analyzed using the PCP Disso v 3.0 software, India.

Preparation of Solid-Self Emulsifying Drug Delivery System (Solid-SEDDS)

Solid-SEDDS was prepared by mixing liquid SEDDS containing CND with Neusilin US2 in $1: 1,1: 2$ and $2: 1$ proportions. In brief liquid SEDDS was added gradually over Neusilin contained in a mortar. After each addition, mixture was mixed vigorously and homogenized to ensure uniform distribution of formulation. Resultant damp mass was passed through sieve no. 120 and dried at ambient temperature and stored until further use.

Characterization of Solid-SEDDS

Micromeritic Properties of Solid-SEDDS The bulk density, tapped density, Carr's Compressibility Index and Hausner ratio were determined for the optimized solid-SEDDS. The angle of repose of self emulsifying powder was determined by funnel method. ${ }^{22}$ Briefly the sample was poured through a funnel with its tip positioned at a fixed height $(h)$ on a horizontal surface until apex of pile touches the tip of the funnel. The angle of repose was calculated using the formula tan $\theta=h / r$ where $r$ is radius of the pile of powder. Additionally the flow rate was determined by measuring the time required for $1.0 \mathrm{~g}$ of formulation to flow through funnel with orifice of $1.5 \mathrm{~cm}$ diameter. The powder flow property was noted on the basis of time required to pass through orifice as less than $1 \mathrm{~s}$ (excellent), less than $5 \mathrm{~s}$ (good), less than $10 \mathrm{~s}$ (average) and more than $10 \mathrm{~s}$ (poor). ${ }^{23)}$

Morphological Analysis

The outer macroscopic structure of the solid self emulsify- ing powder was investigated by scanning electron microscopy (S-4100, Hitachi, Japan) at $15 \mathrm{keV}$ accelerating voltage.

Differential Scanning Calorimetry (DSC)

The physical state of the CND in solid-SEDDS was characterized by DSC studies. The DSC thermograms of the CND, physical mixture of CND with Neusilin as well as that of solid-SEDDS were recorded using differential scanning calorimeter (PerkinElmer, Inc., U.S.A.). About $2 \mathrm{mg}$ of the samples were heated in an open aluminum pan from 30 to $450^{\circ} \mathrm{C}$ at a scanning rate of $10^{\circ} \mathrm{C} / \mathrm{min}$ under the stream of nitrogen.

X-Ray Powder Diffraction Studies

$\mathrm{X}$-Ray powder scattering measurements of the CND, physical mixture of CND with Neusilin and that of solid self emulsifying powder were carried out with X-ray diffractometer (X'Pert PRO PANalytical, U.S.A.). About $1 \mathrm{~g}$ of the sample was required for the analysis. The X-ray powder diffraction patterns were recorded at room temperature using monochromatic $\mathrm{CuK \alpha 1}$ radiation $(k=1.5406 \AA)$ at $40 \mathrm{~mA}$ and at $45 \mathrm{kV}$ over a range of $2 \theta$ angles from $3^{\circ}$ to $50^{\circ}$ with an angular increment of $.02^{\circ}$ per second.

Emulsion Droplet Size

The SMD and PDI of solid-SEDDS was determined by photon correlation spectroscopy using nanosizer (Nanophox NX0088, Sympatec Germany). The formulation was diluted with filtered $(0.45 \mu \mathrm{m}$, Millipore) double distilled water before analysis. Size analysis was carried at $25^{\circ} \mathrm{C}$ with an angle of detection of $90^{\circ}$.

\section{Drug Content Estimation}

Liquid SEDDS and solid-SEDDS containing CND, each equivalent to $10 \mathrm{mg}$ was dispersed in suitable quantity of methanol. The samples were mixed thoroughly to dissolve the drug in methanol, centrifuged at $3000 \mathrm{rpm}$ for $15 \mathrm{~min}$ using 12C micro-centrifuge (Remi Motors, Mumbai, India) to separate the undissolved excipients. The supernatant was suitably diluted and analyzed spectrophotometrically at $240 \mathrm{~nm}$ using Jasco UV-Vis spectrophotometer.

\section{In Vitro Dissolution Studies}

Drug dissolution from solid-SEDDS was performed using USP type II dissolution apparatus (Electrolab, TDT-06 T Mumbai, India). The medium for dissolution studies was $900 \mathrm{~mL}$ of $\mathrm{pH} 1.2$ buffer with paddle rotation speed of $100 \mathrm{rpm}$. An aliquot of $5 \mathrm{~mL}$ was withdrawn at predetermined time intervals and filtered through $0.45 \mu \mathrm{m}$ filter. The concentration of CND in the filtrate was analyzed using UV spectrophotometer at $240 \mathrm{~nm}$ after suitable dilution. Similarly the dissolution studies were performed for pure drug and marketed formulation of the drug.

Calculation of Dissolution Parameters

Various dissolution parameters like dissolution efficiency (D. $E_{15}$ ) and mean dissolution time (MDT) were calculated. ${ }^{24}$ The D.E is defined as the area under the dissolution curve up to a certain time $(t)$, expressed as a percentage of the area of the rectangle described by $100 \%$ dissolution in the same time. D.E can be calculated by using the following equation:

$$
\text { D.E }=\frac{\int_{0}^{t} y \times \mathrm{d} t}{y_{100} \times t} 100 \%
$$

where $y$ is the drug percent dissolved at time $t$.

The MDT can be calculated by the following expression: 


$$
\mathrm{MDT}=\frac{\sum_{i=1}^{n} t_{\mathrm{mid}} \Delta M}{\sum_{i=1}^{n} \Delta M}
$$

where $i=$ dissolution sample number, $n=$ total number of dissolution sample time, $t_{\text {mid }}$ the time at midpoint between $t_{i}$ and $t_{i-1}$, and $\Delta M=$ additional amount of drug dissolved between $t_{i}$ and $t_{i-1}$.

\section{In Vitro Absorption Study}

In vitro absorption study of pure drug, liquid SEDDS and solid-SEDDS were carried out through everted rat intestinal segment using an in-house fabricated perfusion apparatus. ${ }^{25)}$ The apparatus consists of two cylindrical glass tubes; one joined to other via J-shaped tapering end. Both the tubes are held together by a glass joint on the upper end. On the lower ends of both tubes a bulge is given for proper mounting of tissue. After mounting the everted intestinal segment on the apparatus and setting it in the beaker; the inside of the glass tubes serve as the serosal compartment and the beaker serves as the mucosal compartment. For experimental purpose the rat was sacrificed humanely by cervical dislocation and the abdomen was opened by midline incision. A $9 \mathrm{~cm}$ intestinal segment corresponding to duodenal region was carefully removed and transferred to a petri dish containing Kreb's medium (118.0 mM NaCl, $4.7 \mathrm{~mm} \mathrm{KCl}, 2.5 \mathrm{~mm} \mathrm{CaCl}_{2}, 1.2 \mathrm{~mm}$ $\mathrm{MgSO}_{4} \cdot 7 \mathrm{H}_{2} \mathrm{O}, 25.0 \mathrm{~mm} \mathrm{NaHCO}_{3}, 1.2 \mathrm{~mm} \mathrm{KH}_{2} \mathrm{PO} 4$ and $5.5 \mathrm{~mm}$ glucose). The intestinal segment was cleaned with the Kreb's solution and gently everted using a glass rod. A $6.0 \mathrm{~cm}$ everted segment was then mounted in the apparatus which was placed in a $600.0 \mathrm{~mL}$ beaker containing the drug suspended in $500 \mathrm{~mL}$ of pH 5.8 buffer solution. This assembly (beaker and apparatus with tissue) was placed on a magnetic stirrer and a magnetic bead was allowed to rotate at $25 \mathrm{rpm}$ in beaker and the temperature was maintained at $37 \pm 0.5^{\circ} \mathrm{C}$ with adequate aeration. The drug diffused from phosphate buffer $\mathrm{pH} 5.8$ (mucosal side) to the Kreb's solution contained in the tubes (serosal side). The samples were collected at different time points (at every $15 \mathrm{~min}$ for $2 \mathrm{~h}$ ) from the serosal compartment

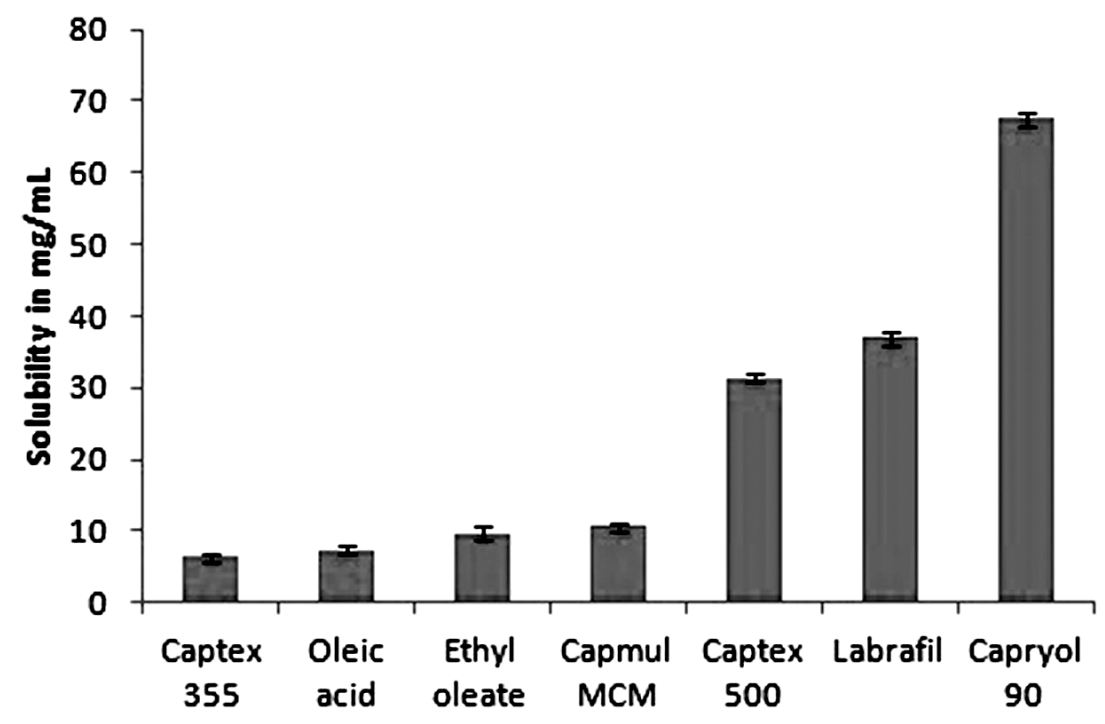

Fig. 1. Solubility Studies of Cilnidipine in Various Oils

Data expressed as mean \pm S.D., $n=3$.

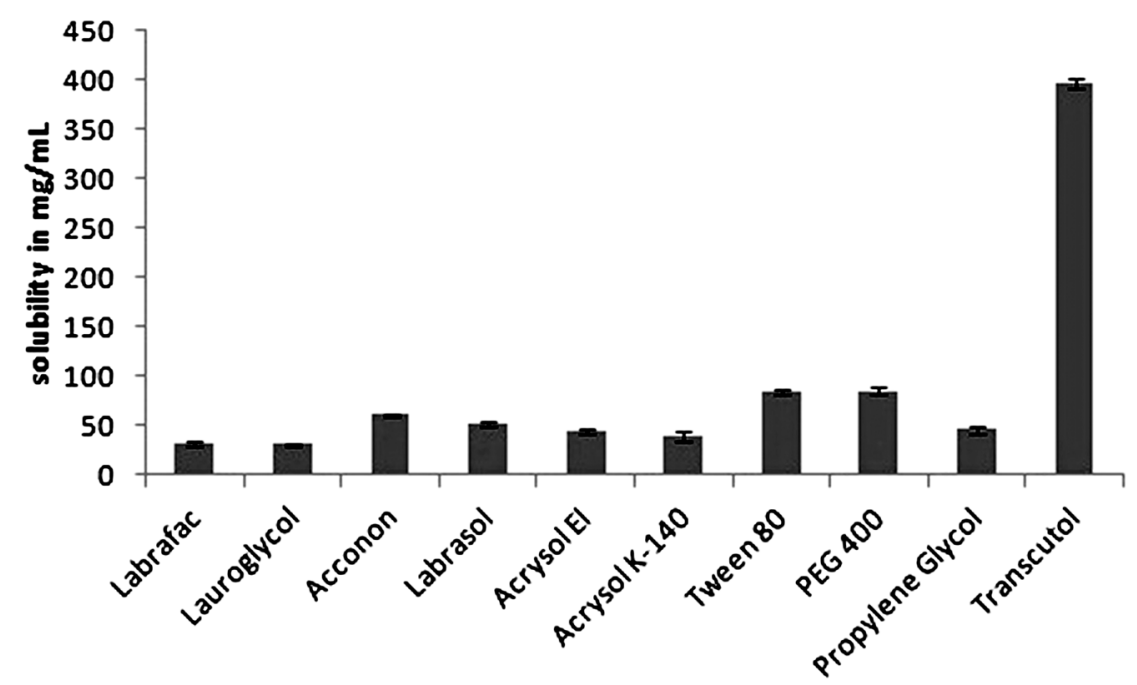

Fig. 2. Solubility Studies of Cilnidipine in Various Surfactants and Co-surfactants

Data expressed as mean \pm S.D., $n=3$. 
and analyzed for the drug content by UV spectrophotometer. Similarly the absorption studies were carried out for the optimized liquid SEDDS and solid-SEDDS. All the experiments were performed in triplicate. The protocol for in vitro absorption study was approved by the Institutional Animal Ethics Committee (IAEC) of Department of Pharmaceutical Sciences, Nagpur and is in accordance with guidance of Committee for the purpose of Control and Supervision of Experiments on Animals (CPCSEA), Ministry of Social Justice and Empowerment, Government of India.

Statistical Analysis All the results were expressed as mean with standard deviation (mean \pm S.D.). The in vitro release data were analyzed by one-way ANOVA using Turkey's $t$-test. The dissolution data obtained was subjected to one-way ANOVA followed by Dunnett's multiple comparison test. The difference at $p<0.05$ was considered to be statistically significant. The statistical analysis was performed with Instant Graph Pad Prism software (version 4.00; Graph Pad Software, San Diego CA, U.S.A.).

\section{Results and Discussion}

Solubility Studies The solubility study of drug in lipid excipients is an important prerequisite in the development of a stable SEDDS with efficient drug loading ability. Identifying the suitable oil, surfactant/co-surfactant having maximal solubilizing potential for drug under investigation not only achieves optimum drug loading but also minimizes the final volume of SEDDS. ${ }^{26,27)}$ Thus the solubility of CND was adjudged in variety of oils, surfactants and co-surfactants. The data is presented in the Figs. 1 and 2. Amongst all the oils screened, CND showed maximum solubilization in Capryol $90(67.6 \pm 0.577 \mathrm{mg} / \mathrm{mL})$ followed by Labrafil $(37.0 \pm 1 \mathrm{mg} / \mathrm{mL})$. Least solubility was observed with Captex 355 as the oil phase $(6.3 \pm 0.57 \mathrm{mg} / \mathrm{mL})$. From the data obtained from the solubility studies Capryol 90 was selected as oil phase for further studies. The surfactant and the co-surfactant were selected based on two parameters, ability to solubilize CND and their emulsification ability.

Preliminary Screening of Surfactants Turbidimetry studies were performed to evaluate the ability of various surfactants to emulsify the selected oil phase, Capryol 90. For oil-surfactant mixture to be used in SEDDS formulations it was essential to determine whether it could disperse efficiently to form spontaneous microemulsion. ${ }^{28)}$ Tween 80, Labrasol and Acrysol EL 135 were selected for the emulsification study as they showed good solubility potential for CND. Thus when Capryol 90 was mixed with the selected surfactants in $1: 1$ ratio and the resulting mixture, $50 \mathrm{mg}$ was diluted to $50 \mathrm{~mL}$ with water, the $\%$ transmittance values of the various dispersions were obtained as $97.7 \pm 0.61,84.3 \pm 1.13$ and $85.4 \pm 0.99$ with Tween 80, Labrasol and Acrysol EL 135 as the surfactants respectively. The study clearly distinguished the ability of the surfactants in emulsifying the selected oil phase and indicated the good emulsifying ability of Tween 80 among all the surfactants screened. Tween 80 was therefore selected as the surfactant for further investigations.

Preliminary Screening of Co-surfactants Addition of a co-surfactant to surfactant containing formulation is reported to improve the emulsification ability of the surfactant and drug absorption from the formulation. ${ }^{29)}$ In the present study Transcutol HP, PEG 400 and propylene glycol were investi- gated as the co-surfactants for improving the emulsification ability of Tween 80 . The $\%$ transmittance values of various dispersions were found to be $99.3 \pm 0.55,98.9 \pm 0.18,87.3 \pm 1.74$ with Transcutol HP, PEG 400 and propylene glycol respectively as the co-surfactants. It was evident from the observations that both Transcutol HP and PEG 400 appeared to be equivalent in emulsifying Capryol 90 when combined with
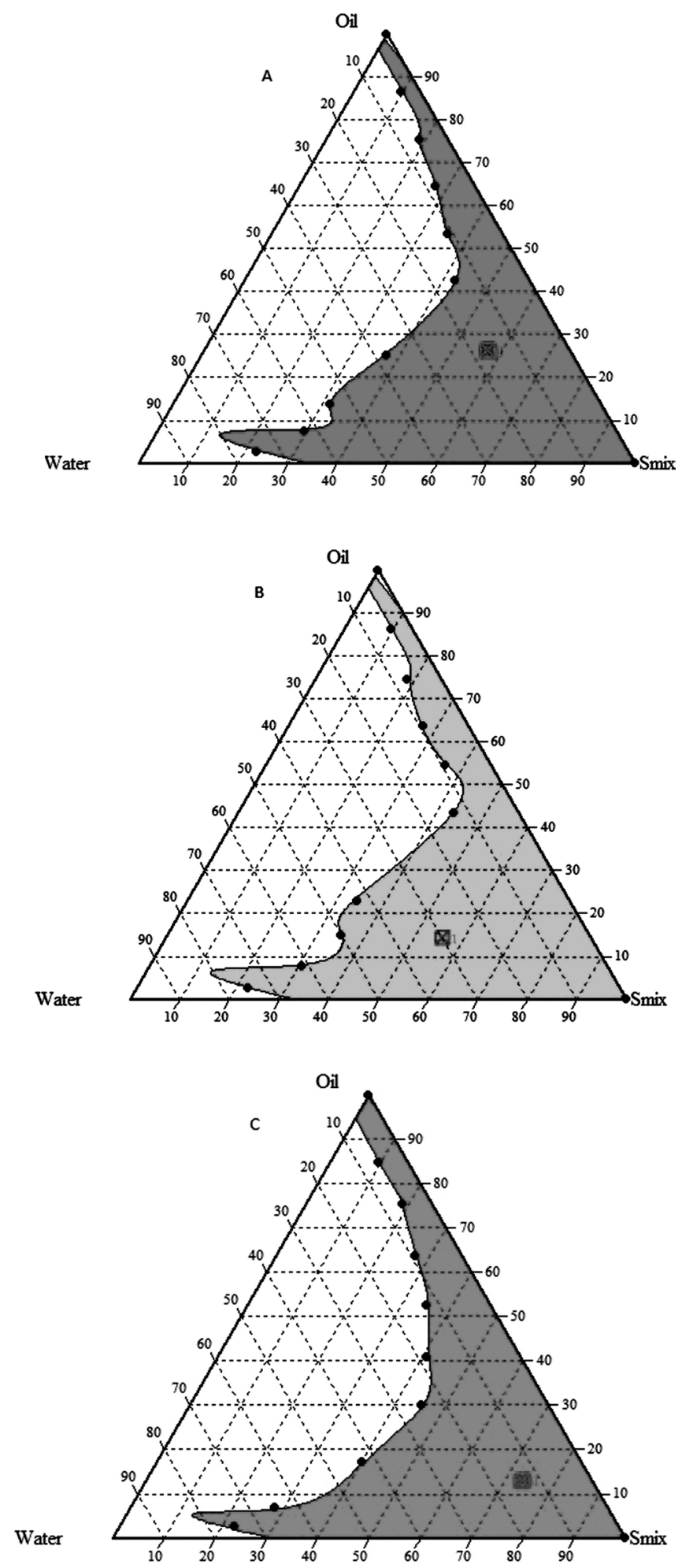

Fig. 3. Pseudoternary Phase Diagrams Involving Capryol 90 (Oil), Tween $80+$ Transcutol HP $\left(S_{\text {mix }}\right)$ and Water

Ratio of surfactant to co-surfactant in (A) is $1: 1$, in (B) is $2: 1$ and in (C) is $1: 2$. 
Tween 80 as compared to propylene glycol. However giving due consideration to the data of the solubility studies and the well known potential of Transcutol HP as a bioavailability enhancer it was deemed fit to be desired co-surfactant for formulation purpose.

Construction of Pseudoternary Phase Diagrams Pseudoternary phase diagrams were constructed as depicted in Figs. $3 \mathrm{~A}-\mathrm{C}$ to identify the microemulsion regions and to optimize the concentration of selected vehicles. For the development of SEDDS formulations optimum ratios of excipients concentrations established by means of phase diagram studies provided the area of monophasic region. It is important to determine this area in order to ensure successful aqueous dilution without breaking the nanoemulsions. ${ }^{30)}$ In the present study the phase diagrams were plotted taking three ratios of surfactant/co-surfactant as $1: 1,2: 1$ and $1: 2$. It was observed that the area of nanoemulsion existence was higher at surfactant/co-surfactant ratio of $2: 1$ and $1: 1$ as compared to that of $1: 2$. Hence for the formulation of SEDDS the ratio of surfactant/co-surfactant was kept at 1:1,2:1 and higher.

Preparation of Liquid SEDDS Twelve batches of liquid SEDDS were prepared as per the composition depicted in Table 1 which was then characterized for various parameters as shown in the same table.

Characterization of SEDDS The liquid SEDDS formulations (F1-F12) were subjected to assessment of efficiency of self-emulsification visually. It is imperative that the SEDDS should disperse completely and quickly on being diluted under mild agitation. In the present investigations, the self emulsification time of all the SEDDS formulations containing $20 \%$ and $30 \%$ of the oil phase were found to be below $60 \mathrm{~s}$ indicating the rapidity of emulsion formations. However formulations containing $40 \%$ of the oil phase had self emulsification time greater than $60 \mathrm{~s}$ suggesting increase in the oil content led to a decrease in emulsification ability. Formulation F6 on constitution with distilled water formed a clear, transparent emulsion which can be termed as Grade A emulsion while remaining formulations containing $20 \%$ and $30 \%$ of the oil phase were observed to form translucent emulsions which can be categorized as Grade B emulsions. However formulations with $40 \%$ of the oil content formed turbid, whitish emulsions which can be termed as Grade $\mathrm{C}$ emulsions.

After being administered orally SEDDS are expected to undergo gradual dilution in contact with GI fluid. A well formulated SEDDS will disperse effectively to form uniform emulsions at different dilutions without precipitation of drug which in turn will ensure uniform release of drug. Hence, robustness to dilution was monitored by diluting the SEDDS 50, 100, 250 and 1000 times with distilled water and with $\mathrm{pH}$ 1.2 and $\mathrm{pH} 6.8$ buffer. It was observed that all the resulting emulsions were found to be stable proving their robustness to dilution. This also indicates the probability of uniform drug release profile 'in vivo' where the formulation is expected to encounter gradual dilution. Even after $24 \mathrm{~h}$, neither precipitation of the drug nor any phase separation was observed when the SEDDS was diluted up to 1000 times, showing the stability of the constituted emulsion.

The liquid SEDDS formulations were characterized for $\%$ transmittance and droplet size. It was revealed that the $\%$ transmittance of F6 was highest among all the formulations indicating a good clarity of the microemulsion being formed which was confirmed by the resultant average droplet size of the formulation. The droplet size of the emulsion is a crucial factor in self-emulsification performance because it determines the rate and extent of drug release as well as absorbance. ${ }^{31)}$ Droplet size of formulations F3, F4 and F6 at 100 times dilution were found to below $200 \mathrm{~nm}$ while those of formulations F1, F2, F8 and F9 were between 200-250 nm. However formulations containing $40 \%$ of the oil phase formed turbid emulsions on being diluted with water with \% transmittance values less than $70 \%$. The average droplet size of formulations with $40 \%$ of the oil phase was determined to be in the range of $355-500 \mathrm{~nm}$. The PDI of most of the formulations was found to be $<0.3$ indicating homogenous distribution of the oil globules.

The cloud point is a vital parameter in assessing the stability of the SEDDS formulations. The cloud point is the temperature above which the clarity of the formulation turns to cloudiness. This happens due to phase separation and precipitation of the drug from the microemulsions formed from the SEDDS which in turn will obviously hamper the drug release. To avoid this phenomenon the cloud point for SEDDS should be above body temperature $\left(37^{\circ} \mathrm{C}\right)$. In the present case the cloud point temperatures of different formulations determined were in the range of $78-84^{\circ} \mathrm{C}$. This infers not only good thermal stability of all the tested formulations but also gives an

Table 1. Formulation and Characterization of SEDDS

\begin{tabular}{|c|c|c|c|c|c|c|c|}
\hline Batch & $\mathrm{X}$ & $\mathrm{Y}$ & $\mathrm{Z}$ & SET* & $\% \mathrm{TM}^{*}$ & Avg. size* & PDI* \\
\hline $\mathrm{F} 1$ & 20 & 70 & 10 & $31 \pm 1.5$ & $83.6 \pm 0.81$ & $243.3 \pm 3.8$ & $0.23 \pm 0.014$ \\
\hline $\mathrm{F} 2$ & 20 & 60 & 20 & $39 \pm 1.5$ & $85.4 \pm 1.2$ & $229.6 \pm 1.8$ & $0.241 \pm 0.016$ \\
\hline F3 & 20 & 50 & 30 & $47 \pm 4.3$ & $92.7 \pm 1.5$ & $175.6 \pm 4.5$ & $0.325 \pm 0.021$ \\
\hline F4 & 20 & 40 & 40 & $31 \pm 1$ & $90.5 \pm 1.3$ & $181.3 \pm 5.8$ & $0.252 \pm 0.015$ \\
\hline F5 & 20 & 30 & 50 & $51 \pm 1.1$ & $72.1 \pm 1.0$ & $293.3 \pm 4.7$ & $0.237 \pm 0.066$ \\
\hline F6 & 30 & 60 & 10 & $31 \pm 1$ & $98.1 \pm 1.1$ & $137.0 \pm 2.0$ & $0.287 \pm 0.014$ \\
\hline F7 & 30 & 50 & 20 & $48 \pm 1.1$ & $81.5 \pm 1.3$ & $262.6 \pm 5.1$ & $0.238 \pm 0.100$ \\
\hline F8 & 30 & 40 & 30 & $29 \pm 1.1$ & $88.6 \pm 1.6$ & $209.6 \pm 4.9$ & $0.257 \pm 0.036$ \\
\hline F9 & 30 & 30 & 40 & $41 \pm 1$ & $85.6 \pm 1.5$ & $243.3 \pm 3.6$ & $0.238 \pm 0.019$ \\
\hline F10 & 40 & 50 & 10 & $84 \pm 5.0$ & $68.5 \pm 1.7$ & $413.8 \pm 1.8$ & $0.314 \pm 0.103$ \\
\hline F11 & 40 & 40 & 20 & $90 \pm 5.5$ & $72.6 \pm 2.2$ & $355 \pm 8.8$ & $0.333 \pm 0.103$ \\
\hline F12 & 40 & 30 & 30 & $84.6 \pm 6.1$ & $60.7 \pm 2.5$ & $500.8 \pm 8.8$ & $0.282 \pm 0.045$ \\
\hline
\end{tabular}

X: \% Oil, Y: \% Surfactant, Z: \% Co-surfactant, SET: Self emulsification time in seconds, \%TM: \% Transmittance, PDI: Polydispersity index. * Data expressed as mean \pm S.D. $(n=3)$. 
indication of unhindered drug release.

Thermodynamic stability indicates the kinetic stability of a formulation and is employed to study the chemical reaction between the excipients of a formulation. The SEDDS have the ability to form a spontaneous microemulsion in the GIT with mild agitation provided by the GI fluid. As such formulation should possess considerable stability in order to prevent precipitation, creaming or cracking. Hence the formulations were subjected to heating and cooling cycle exposure as well as to centrifugation studies. The observations revealed that emulsions could withstand wide range of temperature changes and centrifugal stress without any phase separation and drug precipitation.

Conventional dissolution testing of SEDDS has a limitation in mimicking its real time in vivo dissolution and such a technique can only provide a measure of dispersibility of SEDDS in the dissolution medium. ${ }^{32}$ After oral administration when SEDDS encounter aqueous medium, drug may present in free molecular state or in emulsion form or in solubilized micellar solution. ${ }^{33)}$ In order to assess the real drug release pattern of formulation drug dissolved in aqueous medium should be separated from emulsion associated portion of the drug. For this purpose dialysis bag method was employed to facilitate the actual drug release pattern. The formulations were diluted with $\mathrm{pH} 1.2$ buffers to circumvent the sticking of formulation with the membrane as reported. ${ }^{34)}$ Four formulations were selected for drug release studies having droplet sizes below and close to $200 \mathrm{~nm}$. Thus Fig. 4 shows the in vitro drug release profiles of the liquid SEDDS formulations F3, F4, F6 and F8 over a period of $180 \mathrm{~min}$. Among those, F6 formulation showed highest drug release $(98.2 \%)$ compared to other formulations. Statistical analysis of drug release with one-way ANOVA followed by Turkey test revealed significant enhanced release from formulation F6 in comparison to formulations F3, F4 and F8 $(p<0.05)$. This could be due to proper composition of oil and surfactant in the self emulsifying system of F6 resulting in lower droplet size $(137 \mathrm{~nm})$ and higher surface area for drug partitioning which eventually equilibrated with the dialyzing medium. The drug release pattern clearly indicated the effect of average droplet size on drug diffusion across dialyzing membrane. Similar observations have been reported earlier. $^{35)}$

Based on the aforementioned results of various parameters the optimized formulation selected for preparation of solid-

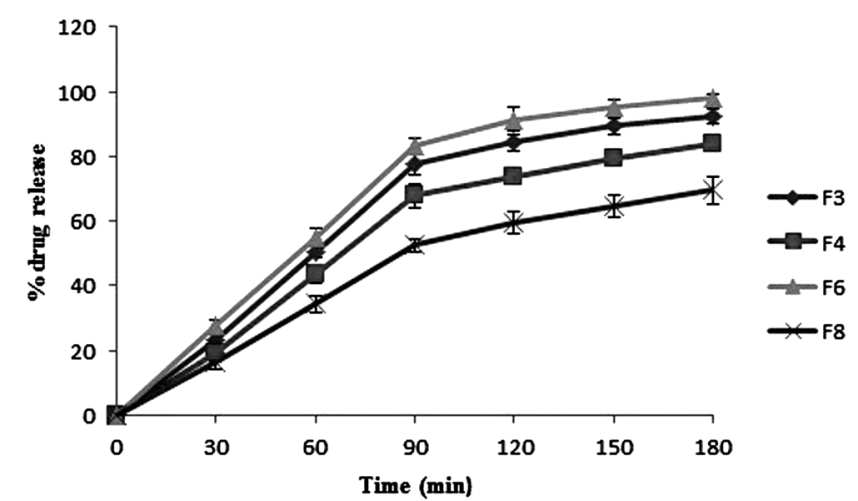

Fig. 4. In Vitro Release Profiles of Cilnidipine from SEDDS Formulations

Mean \pm S.D., $n=3$.
SEDDS was formulation F6 since it was found to give the least droplet size, highest drug release along with other desirable physicochemical properties. Ten milligram of CND was loaded to the optimized formulation at a ratio of $1: 13$.

Preparation of Solid Self-emulsifying Drug Delivery System (Solid-SEDDS) One of the major aspect in the development of solid-SEDDS is to find the right carrier that will carry the adequate lipid load and at the same time exhibit good powder flow properties desirable for the development of a solid dosage form. Liquid SEDDS containing CND were adsorbed onto Neusilin US2 in $1: 1,1: 2$ and $2: 1$ proportions. Incorporation of liquid SEDDS with Neusilin US2 at $1: 1$ $\mathrm{w} / \mathrm{w}$ ratio resulted in free flowing dry powder as compared to $2: 1$ ratio. The appearance of the powder was such that one would have difficulty to recognize visually that the adsorbent contained an equal weight of oily liquid. Thus $1: 1$ proportion of liquid SEDDS to Neusilin US2 was considered as the most appropriate ratio for formulating solid-SEDDS of CND by adsorption technique.

Micromeritic Properties of Solid-SEDDS The bulk and tap density of the powder was $0.2834 \pm 0.0129$ and $0.3378 \pm 0.0145 \mathrm{~g} / \mathrm{cc}$, respectively as depicted in Table 2 . The compressibility index (CI), which is also known as the Carr's Index, and the Hausner ratio (HR), which is essentially the ratio of the bulk density to the tap density of powders are indirectly influenced by bulk density, tap density, size, shape, moisture content and cohesiveness of the materials and, therefore, serve as useful parameters to assess powder properties. In the present investigation, the CI and HR value of the prepared powder was found to be $11.03 \pm 0.04$ and1.16 \pm 0.002 , respectively and thus can be termed as "good" according to the USP classification system, ${ }^{36)}$ indicating that there would not be any problem with the flow of powders. The angle of repose is an old and simple technique that also gives a general idea of the cohesivity and flow properties of powder; however, it is heavily affected by the methodology of the test and may not be highly reproducible. ${ }^{37)}$ Nevertheless the angle of repose of the formulated powder was determined in the present case and the results showed 'excellent' values as per the USP guideline. Similarly the flow rate of the powder was found to be less than $1 \mathrm{~s}$ substantiating the good flow property of the powder.

Morphological Analysis The scanning electron micrographs in Fig. 5 revealed CND as crystalline powder with rectangular shaped crystals. The carrier Neusilin US2 appeared as granular and highly porous material with relatively large pores. The self emulsifying powder showed presence of smooth granular particles and free from rough edges. No distinct crystals were evident on the surface of the particles after adsorbing the liquid SEDDS on the surface of Neusilin indicating that the adsorbed liquids are localized or lodged

Table 2. Micromeritic Properties of Solid-SEDDS

\begin{tabular}{lc}
\hline \hline Properties of solid-SEDDS & Results \\
\hline Bulk density $(\mathrm{g} / \mathrm{cc})^{*}$ & $0.2834 \pm 0.0129$ \\
Tap density $(\mathrm{g} / \mathrm{cc})^{*}$ & $0.3378 \pm 0.0145$ \\
Carr's compressibility index* & $11.03 \pm 0.04$ \\
Hausner ratio* & $1.16 \pm 0.002$ \\
Angle of repose (degree)* & $27.63 \pm 0.04$ \\
Flow rate* & $<1 \mathrm{~s}$ \\
\hline
\end{tabular}

*Values expressed as mean \pm S.D. $(n=3)$ 

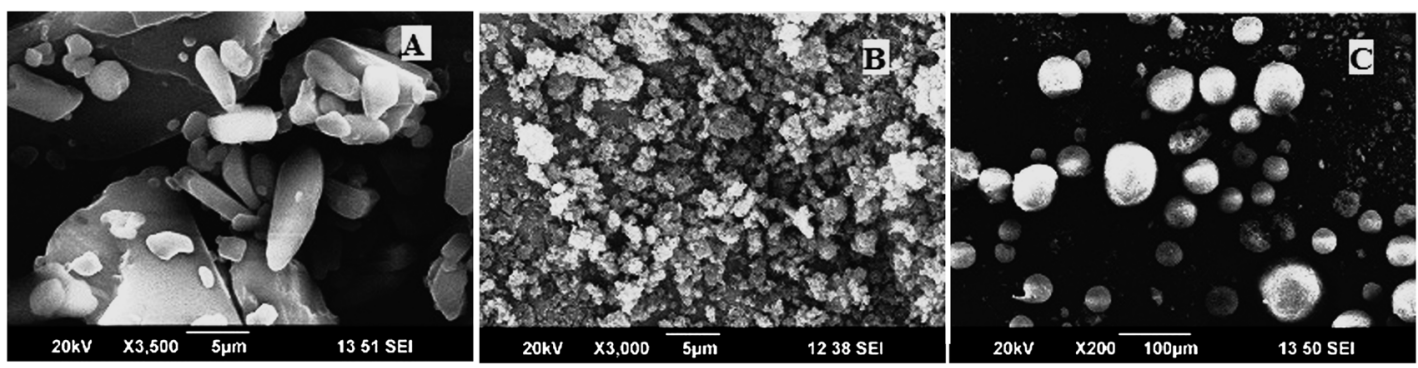

Fig. 5. Scanning Electron Micrographs of (A) Pure Drug (B) Neusilin US2 and (C) Solid-SEDDS

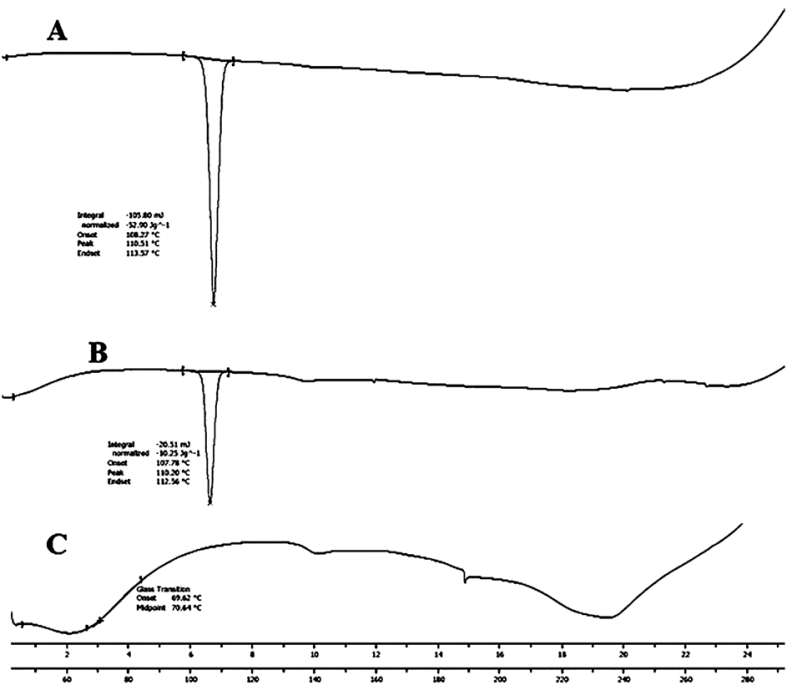

Fig. 6. DSC Thermograms of (A) Cilnidipine (B) Physical Mixture of Cilnidipine and Neusilin and (C) Solid-SEDDS

deep inside the pores of the spherical particles, leaving very little liquids on the surface of particles. The particles of solidSEDDS were observed to be spherical as evident from the SEM images indicating a good flow property which will be essential for transformation of liquid SEDDS into an appropriate solid dosage form.

DSC DSC allows determination of thermotropic phase transition behavior in a quantitative manner. The thermograms recorded during analysis display pronounced melting peaks. The narrow peak at $110.51^{\circ} \mathrm{C}$ for pure CND (Fig. 6) infers presence of crystalline form of the drug. The physical mixture of the drug and the carrier retained the crystalline peak of the drug but with reduced intensity. No representative peaks for drug were observed for solid SEDDS indicating the possibility of transformation of crystalline form of the drug to the amorphous one.

X-Ray Powder Diffraction Studies The X-ray powder diffractograms of CND, physical mixture of CND and Neusilin as well as that of solid-SEDDS is shown in Fig. 7. The diffraction pattern of CND showed characteristics sharp peaks at numerous 2 theta values of 5.956, 11.920, 14.382, 16.581, $18.811,21.867,23.344$ and 25.030 respectively indicating the presence of crystalline form of the drug (Fig. 7A). Most of the characteristics peaks of Cilnidipine were observed in the diffraction pattern of physical mixture of CND and Neusilin (Fig. 7B) indicating that the drug was still present in the crystalline form. The absence of peaks of the drug in the diffractograms of solid-SEDDS (Fig. 7C) implied that the drug
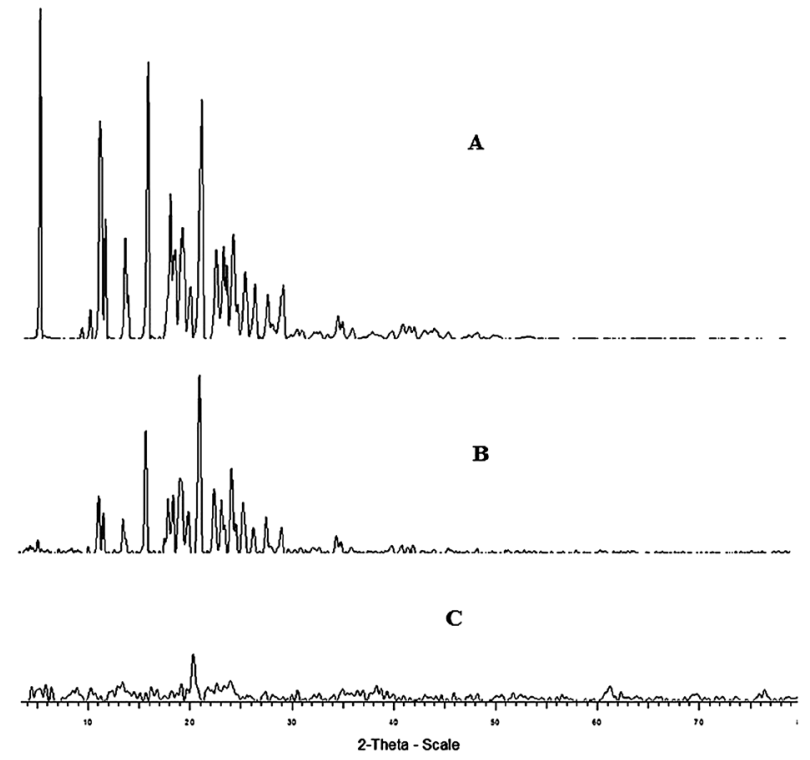

Fig. 7. X-Ray Powder Diffraction Spectra of (A) Cilnidipine (B) Physical Mixture of Drug and Neusilin and (C) Solid-SEDDS

was no longer present in the crystalline form when prepared as solid-SEDDS formulation suggesting the transformation of crystalline form of the drug to amorphous form. This transformation may have contributed to faster dissolution rate of solid-SEDDS as the material in its amorphous form dissolves at a faster rate due to its higher internal energy and molecular motion when compared to crystalline forms. ${ }^{38)}$

Emulsion Droplet Size The average droplet size of the liquid and the solid-SEDDS was found to less than $200 \mathrm{~nm}$ with PDI values $<0.3$ (Fig. 8). There was no significant difference in the average droplet size of both the system suggesting that even after solidification the system is able to retain its droplet size and has the probability of releasing the drug in the more or less the same manner in vitro as well as in vivo.

Drug Content Estimation The drug content of liquid SEDDS (F6) and solid-SEDDS was found to $99.65 \pm 0.312 \%$ and $98.72 \pm 0.063 \%$ respectively. The data proved that the particles of Neusilin had effectively adsorbed the liquid SEDDS formulation due to their highly porous nature and presence of larger interparticulate void spaces.

In Vitro Dissolution Studies Dissolution profile of pure drug, marketed formulation and solid-SEDDS were carried out under standard conditions in $\mathrm{pH} 1.2$ buffer (Fig. 9). The drug dissolution studies portrayed optimized solid-SEDDS formulations exhibited faster drug dissolution upto $92.3 \pm 2.8 \%$

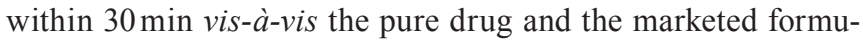



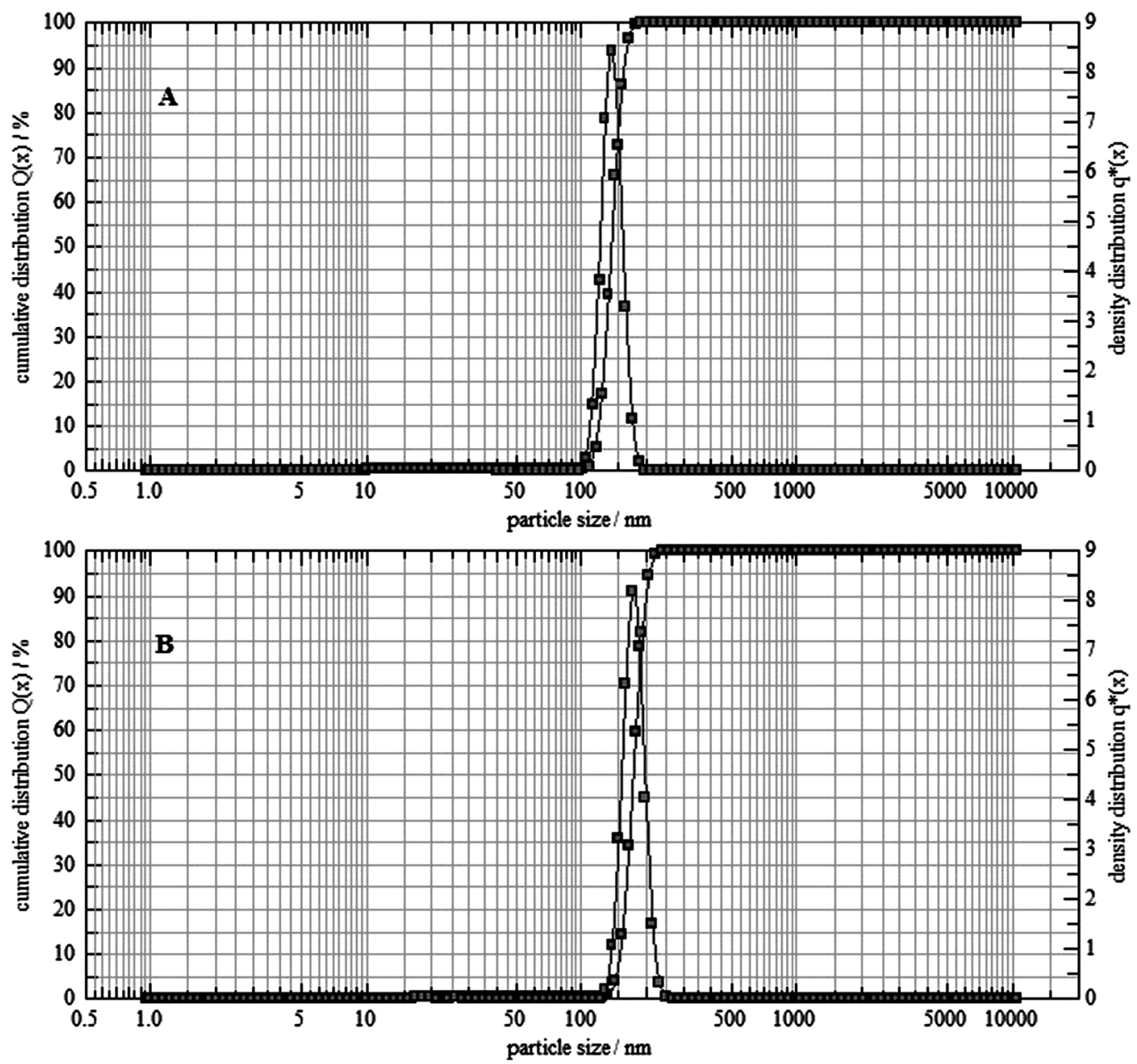

Fig. 8. Droplet Size Distribution of (A) Optimized Liquid SEDDS, F6 and (B) Solid-SEDDS

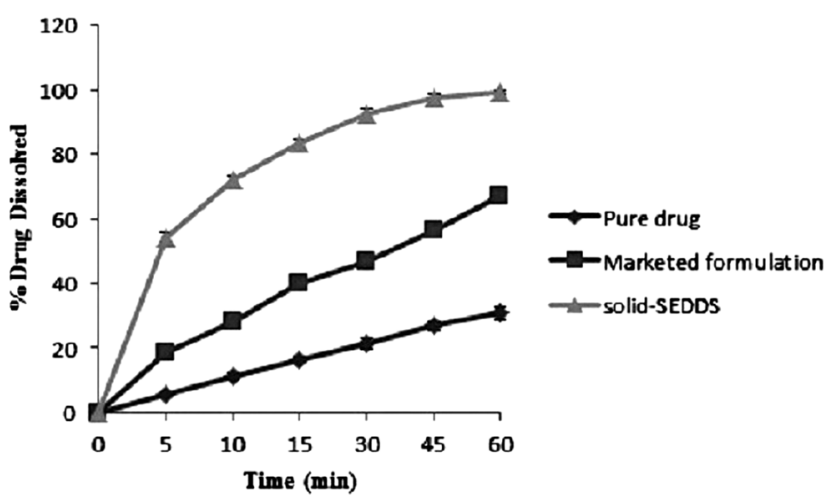

Fig. 9. In Vitro Dissolution Profiles of Pure Drug, Marketed Formulation and Solid-SEDDS

Mean \pm S.D., $n=3$.

lation showed maximum drug dissolution up to $21.3 \pm 1.6 \%$ and $46.5 \pm 1.06 \%$ in $30 \mathrm{~min}$ respectively. Thus the dissolution of the drug was significantly higher from the solid-SEDDS formulation as compared to pure drug and the marketed formulation $(p<0.0001)$. The dissolution parameters compared for the various formulations are as shown in the Table 3 . It is evident that the solid-SEDDS have given higher dissolution efficiency values and lower MDT as compared to the pure drug and the
Table 3. Dissolution Parameters of Solid-SEDDS Formulation along with Pure Drug and the Marketed Formulation (Mean \pm S.D., $n=3$ )

\begin{tabular}{lcc}
\hline \hline Formulation & $\begin{array}{c}\text { Dissolution efficiency } \\
\left({\mathrm{D} . \mathrm{E}_{15}}\right)\end{array}$ & $\begin{array}{c}\text { Mean dissolution time } \\
(\mathrm{MDT})\end{array}$ \\
\hline Pure drug & $8.37 \pm 0.51$ & $7.4 \pm 0.19$ \\
Marketed formulation & $22.25 \pm 0.70$ & $6.7 \pm 0.17$ \\
Solid-SEDDS & $55.99 \pm 1.18 * * *$ & $4.92 \pm 0.04 * * *$ \\
\hline \multirow{2}{*}{ w** $p<0.0001$ when compared with pure drug and marketed formulation using one } \\
\multicolumn{2}{l}{ ANOVA followed by Dunnett's multiple comparison test. }
\end{tabular}

marketed formulation.

In Vitro Absorption Study The absorption profile of the drug from the everted intestinal segment of the rat is shown in the Fig. 10. It was observed that the absorption of the drug was enhanced from liquid SEDDS as $99.9 \pm 3.4 \%$ of the drug was transported from mucosal to serosal side of the rat intestine within $90 \mathrm{~min}$ from SEDDS in comparison to only $42.2 \pm 3.2 \%$ from that of the pure drug suspension. Solid-SEDDS also showed improved drug absorption as $98.7 \pm 2.4 \%$ of the drug was transported within $120 \mathrm{~min}$ although the absorption profile was little slower as compared to the liquid SEDDS. Thus it can be inferred that absorption of the drug from the solidSEDDS has been enhanced and inevitably solid-SEDDS has 


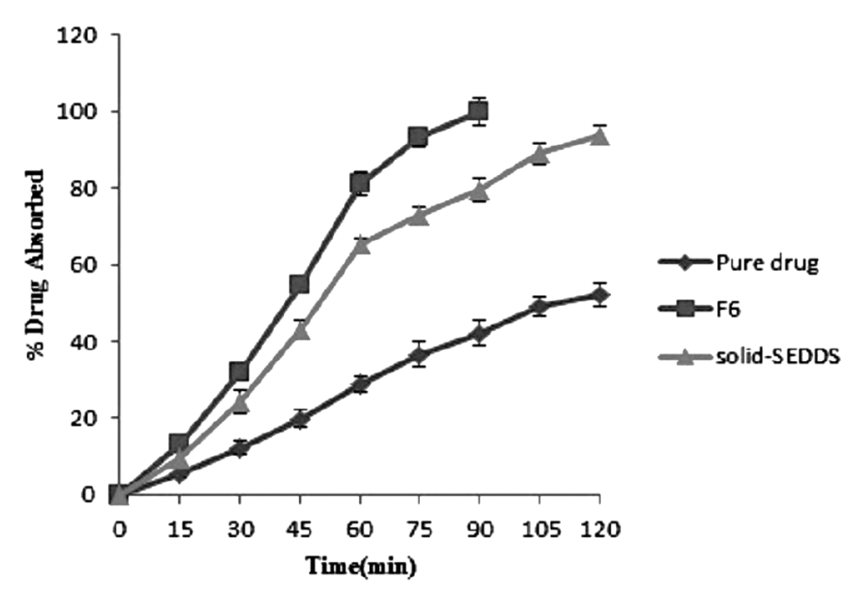

Fig. 10. In Vitro Absorption Profiles of Pure Drug, Optimized Liquid SEDDS and Solid-SEDDS

Mean \pm S.D., $n=3$.

the potential of improved oral delivery of the drug.

\section{Conclusion}

In the current investigations SEDDS of cilnidipine was prepared and evaluated for various parameters. The optimized liquid SEDDS, F6 was successfully transformed into a free flowing powder using Neusilin US2, an effective and excellent porous carrier. The average droplet size of the liquid and solid-SEDDS was less than $200 \mathrm{~nm}$. The enhanced in vitro dissolution and absorption profile from the solid-SEDDS is an indication of improvement in solubility, dissolution rate and bioavailability of the drug. Further investigations are on to develop tablet formulations from solid-SEDDS.

Acknowledgments Authors are thankful to Macleods Pharmaceuticals Limited, Mumbai, India for providing the gift sample of Cilnidipine and to Gattefosse Limited, Mumbai, India, Abitec Corporation, U.S.A. and Corel Pharma Chem, Ahmedabad, Gujarat for providing the gift samples of oils and surfactants. Authors are also grateful to the Sophisticated Analytical Instruments Facility (SAIF), Kochi, India for providing the scanning electron microscopic and powder X-ray diffraction analysis and to the Head, Department of Pharmaceutical Sciences, R.T.M Nagpur University, Nagpur for providing all the necessary facilities and infrastructure for carrying out this study.

Conflict of Interest The authors declare no conflict of interest.

\section{References}

1) Pouton C. W., Eur. J. Pharm. Sci., 29, 278-287 (2006).

2) Mu H., Holm R., Mullertz A., Int. J. Pharm., 453, 215-224 (2013).

3) Pouton C. W., Adv. Drug Deliv. Rev., 25, 47-58 (1997).

4) Charman N. W., Humberstone A. J., Adv. Drug Deliv. Rev., 25, 103-128 (1997).

5) Hauss D. J., Adv. Drug Deliv. Rev., 59, 667-676 (2007).

6) Kohli K., Chopra S., Dhar D., Arora S., Khar R. K., Drug Discov. Today, 15, 958-965 (2010).
7) Tang B., Cheng G., Gu J. C., Xu C. H., Drug Discov. Today, 13, 606-612 (2008).

8) Kang M. J., Jung S. Y., Song W. H., Park J. S., Choi S. U., Oh K. T., Choi H. K., Choi Y. W., Lee J., Lee B. J., Chi S. C., Drug Dev. Ind. Pharm., 37, 1298-1305 (2011).

9) Takahara A., Cardiovas. Ther., 27, 124-139 (2009).

10) Chen C., Xie X., Li Y., Zhou C., Song Y., Yan Z., Yang X., Drug Dev. Ind. Pharm., 40, 441-451 (2014).

11) Hu L., Song W., Niu F., Jiao K., Jia Z., Pak. J. Pharm. Sci., 26, 629-636 (2013).

12) Hu L., Zhang H., Song W., Gu D., Hu Q., Carbohydr. Polym., 90, 1719-1724 (2012).

13) Gumaste S. G., Pawlak S. A., Dalrymple D. M., Nider C. J., Trombetta L. D., Serajuddin A. T. M., Pharm. Res., 30, 3170-3185 (2013).

14) Chaudhari P. P., Bhalerao A. V., Int. J. Pharm. Pharm. Sci., 4, 96-98 (2012).

15) Date A. A., Nagarsenker M. S., Int. J. Pharm., 329, 166-172 (2007).

16) Bachhav Y. G., Patravale V. B., AAPS PharmSciTech, 10, 482-487 (2009).

17) Talegaonkar S., Mustafa G., Akhter S., Iqbal Z. I., J. Disp. Sci. Tech., 31, 690-701 (2010).

18) Balakumar K., Raghavan C. V., Selvan N. T., Prasad R. H., Abdu S., Colloids Surf. B Biointerfaces, 112, 337-343 (2013).

19) Zhang P., Liu Y., Feng N., Xu J., Int. J. Pharm., 355, 269-276 (2008).

20) Shafiq S., Shakeel F., Talegaonkar S., Ahmad F. J., Khar R. K., Ali M., Eur. J. Pharm. Biopharm., 66, 227-243 (2007).

21) Kang B. K., Lee J. S., Chon S. K., Jeong S. Y., Yuk S. H., Khang G., Lee B. H., Cho S. H., Int. J. Pharm., 274, 65-73 (2004).

22) Carr R. L., Chem. Eng., 18, 163-168 (1965).

23) Dixit R. P., Nagarsenker M. S., Eur. J. Pharm. Sci., 35, 183-192 (2008).

24) Costa P., Sousa Lobo J. M., Eur. J. Pharm. Sci., 13, 123-133 (2001).

25) Dixit P., Jain D. K., Dumbwani J., J. Pharmacol. Toxicol. Methods, 65, 13-17 (2012).

26) Lawrence M. J., Rees G. D., Adv. Drug Deliv. Rev., 45, 89-121 (2000).

27) $\overline{\text { Gursoy }}$ R. N., Benita S., Biomed. Pharmacother., 58, 173-182 (2004).

28) Pouton C. W., Porter C. J. H., Adv. Drug Deliv. Rev., 60, 625-637 (2008).

29) Porter C. J. H., Pouton C. W., Cuine J. F., Charman W. N., Adv. Drug Deliv. Rev., 60, 673-691 (2008).

30) Setthacheewakul S., Mahattanadul S., Phadoongsombut N., Pichayakorn W., Wiwattanaptapee R., Eur. J. Pharm. Biopharm., 76, 475-485 (2010).

31) Constantinides P. P., Scalart J. P., Lancaster C., Marcello G., Marks H., Ellens H., Smith P. L., Ellens. Pharm. Res., 11, 1385-1390 (1994).

32) Patil P., Patil V., Paradkar A., Acta Pharm., 57, 111-122 (2007).

33) Kallakunta V. R., Bandari S., Jukanti R., Veerareddy P. R., Powder Technol., 221, 375-382 (2012).

34) Wu W., Wang Y., Que L., Eur. J. Pharm. Biopharm., 63, 288-294 (2006).

35) Goyal U., Arora R., Aggarwal G., Acta Pharm., 62, 357-370 (2012).

36) "Powder Flow 〈1174〉 The United States Pharmacopoeia 30/National Formulary 25 (USP/NF)", United States Pharmacopoeial Convention MD, U.S.A., Rockville (2007).

37) Shah R. B., Tawakkul M. A., Khan M. A., AAPS PharmSciTech, 9, 250-258 (2008).

38) Hancock B. C., Zografi G., J. Pharm. Sci., 86, 1-12 (1997). 